\title{
PRODUCT OF RING VARIETIES AND ATTAINABILITY
}

\author{
BY
}

\author{
AWAD A. ISKANDER
}

ABSTRACT. The class of all rings that are Everett extensions of a ring in a variety $u$ by a ring in a variety $B$ is a variety $u \cdot B$. With respect to this operation the set of all ring varieties is a partially ordered groupoid (under inclusion), that is not associative. A variety is idempotent iff it is the variety of all rings, or generated by a finite number of finite fields. No families of polynomial identities other than those equivalent to $x=x$ or $x=y$ are attainable on the class of all rings or on the class of all commutative rings.

Hanna Neumann [12] introduced the notion of variety product for groups. This product turns the set of all group varieties into a free monoid with zero as shown independently by B. H. Neumann, Hanna Neumann and P. M. Neumann [11], and A. L. Šmilkin [16]. By analogue of groups, A. I. Mal'cev [9] defined the product for classes of algebraic systems and gave several applications. V. A. Parfenov [14] proved that the set of all Lie algebra varieties over a field of characteristic 0 is a free monoid with 0 . However, in rings, the product of varieties is not associative. In the present paper we determine all the idempotent varieties of associative rings. We also apply the results to show that there are no nontrivial sets of identities attainable on the varieties of all associative rings or of all associative and commutative rings; this answers a question of $T$. Tamura [17].

For an account of the variety theory the reader may consult [1], [2], [6], [10], [13].

In this paper we will be concerned only with associative rings not necessarily with 1; the word "ring" will mean "associative ring". German letters will always denote classes or varieties of rings.

The methods of this paper could be modified for the variety of all commutative and associative rings, and the analogue of Theorem 5 holds for the variety of all commutative rings.

1. Definition 1. Let $A$ and $B$ be rings. The ring $C$ is called an extension of $A$ by $B$ if $C$ possesses an ideal isomorphic to $A$ whose factor ring is isomorphic to $B$.

Received by the editors April 2, 1973.

AMS (MOS) subject classifications (1970). Primary 08A15; Secondary 16A06, 16 A38.

Key words and phrases. Associative ring, variety, free ring, T-ideal, polynomial identity, indecomposable, attainable set of identities, Everett extension. 
This notion is due to Everett [4] and is the analogue of Schreier extension of groups [15].

Definition 2. If $\mathscr{A}$ and $B$ are classes of rings $\mathscr{U} . B$ is the class of all rings that are extensions of a ring in $\mathcal{Q}$ by a ring in $B$.

This notion is due to Mal'cev [9] where he proved (for more general systems):

Theorem 1 (Mal'cev [9]). The product of two ring varieties is a ring variety.

Denote the set of all ring varieties by $G$, the variety of all rings by $\cong$ and the variety of all zero (degenerate) rings by $\mathbb{E}$. In [9], it is shown:

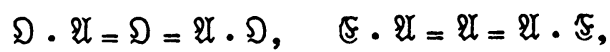

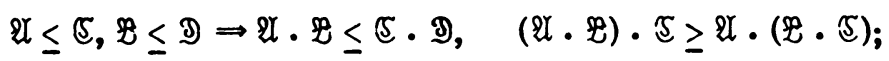

inclusion can be strict.

I.e., $\langle G ; \cdot, \leq\rangle$ is a partially ordered nonassociative groupiod with zero (囚) and a unit (E).

2. Every ring variety is determined by a set of identities of the type $p\left(x_{1}, \ldots, x_{n}\right)=0$. Let $F$ be the free ring over the set of free generators $\left\{x_{1}\right.$, $\left.x_{2}, \ldots\right\}$ (cf. e.g. [3], [13]). If $U \subseteq F$ and $A$ is a ring, let

$$
U(A)=\left\{p\left(a_{1}, \cdots, a_{n}\right): p \in U, a_{1}, \ldots, a_{n} \in A\right\} .
$$

It is well known that $A$ belongs to the variety determined by $U$ iff $U(A)=$ $\bar{U}(A)=0$ where $\bar{U}$ is the $T$-ideal of $F$ generated by $U$ (the smallest ideal of $F$ closed under all endormorphisms of $F$ and containing $U$ ).

Proposition 2. If $U$ is a T-ideal of $F$ and $A$ is a ring, then $U(A)$ is the smallest ideal of $A$ whose factor belongs to $\mathfrak{U}$ the variety determined by $U$.

$$
\begin{aligned}
& a u\left(a_{1}, \ldots, a_{n}\right) \pm v\left(b_{1}, \ldots, b_{m}\right) b=x_{1} u\left(x_{2}, \ldots, x_{n+1}\right) \\
& \quad \pm v\left(x_{n+2}, \cdots, x_{n+m+2}\right) x_{n+m+3}\left(a, a_{1}, \ldots, a_{n}, b_{1}, \ldots, b_{m}, b\right) .
\end{aligned}
$$

Proposition 3 (Mal'cev [9]). Let $U, V$ be two T-ideals of $F$ and let $\mathfrak{U}, \mathfrak{B}$ be the ring varieties determined by $U$ and $V$ respectively. Then $A \in \mathfrak{U} \cdot \mathfrak{B}$ iff $V(A) \in \mathfrak{u}$.

Thus the variety $\mathfrak{U}, \mathfrak{B}$ is determined by the set of identities $U(V)$.

3. For any variety $\mathfrak{U}$ denote by $\delta(\mathfrak{U})$ the least degree [3] of polynomial identities satisfied by $\mathfrak{n} ; \delta(\supseteq)=\infty$.

It is implicit in [5], [13] that the minimal degrees are achieved by homogeneous identities, since if $p_{1}$ is a homogeneous component of $p$, and $p$ is an identity in $\mathfrak{U}$, then $k p_{1}$ is an identity in $\mathfrak{U}$ for some $k \in Z, k>0$. 
For any variety $\mathfrak{U}$ denote by $c(\mathfrak{U})$ the characteristic of the free $\mathfrak{U}$-ring on one generator. Then

Proposition 4. $\mathfrak{U} \leq \mathfrak{B} \Rightarrow \delta(\mathfrak{U}) \leq \delta(\mathfrak{B}), c(\mathfrak{U})=1$ iff $\mathfrak{U}=\mathfrak{E}, c(\mathfrak{U})=0$ iff $\delta(\mathfrak{U})>1$.

Denote by $G F(p, n)$ the Galois field of order $p^{n}$ and by $Q_{p}$ the ring $\left\langle\{0, a, 2 a, 3 a, \ldots,(p-1) a\} ; a^{2}=0\right\rangle$, where $p$ is a prime. The variety generated by $G F(p, n)$ is denoted by $\Re(p, n)$, it is the variety of all rings satisfying: $p x=0, x-x^{p^{n}}=0$. The variety generated by $Q_{p}$ is denoted by $\Re(p)$, it is the variety of all rings satisfying: $p x=0, x y=0$ [19].

4. We now formulate the basic theorem:

Theorem 5. If the variety $\mathfrak{U} \neq \cong$, the following conditions are equivalent:

(i) $\mathfrak{U}$ does not contain any $Q_{p}$.

(ii) $\mathfrak{U}$ is generated by a finite number of finite fields.

(iii) $\mathfrak{U}$ is idempotent in $(G,$.$) , i.e., \mathfrak{a} \cdot \mathfrak{U}=\mathfrak{u}$.

The proof will depend on some lemmas.

Lemma 6. The variety $\mathfrak{B}_{n}$ defined by $p^{n-r} x_{1} x_{2} \cdots x_{2 r}, r=0,1,2, \ldots, n$, is contained in the variety

$$
\Omega(p) \cdot(\Omega(p) \cdot(\ldots(\Omega(p) \cdot \Omega(p)) \ldots))=\Omega(p)^{n} \cdot
$$

Let

$A \in \mathfrak{B}_{n+1^{\circ}} \quad B=\sum p^{n-r} A \ldots A\left(2^{r} A^{\prime}\right.$ s $)$ is an ideal in $A$.

$A \cdot B \in \mathfrak{B}_{n}$.

$$
\begin{gathered}
b \in B \Leftrightarrow b=\sum\left\{p^{n-r} a_{j 1} \cdots a_{j 2^{r}}: 0 \leq r \leq n, j \in K_{r}\right\} . \\
p b=\sum\left\{p^{n+1-r} a_{j 1} \cdots a_{j 2^{r}}: 0 \leq r \leq n, j \in K_{r}\right\}=0 .
\end{gathered}
$$

If $c \in B$ then

$$
\begin{aligned}
b \cdot c= & \left(\sum\left\{p^{n-r} a_{j 1} \cdots a_{j 2^{r}}: 1 \leq r \leq n, j \in K_{r}\right\}\right) \\
& \cdot\left(\sum\left\{p^{n-s} c_{i 1} \cdots c_{i 2} s: 0 \leq s \leq n, i \in L_{s}\right\}\right) \\
= & \sum\left\{a_{j 1} \cdots a_{j 2^{n}} c_{i 1} \cdots c_{i 2^{n}}: j \in K_{n}, i \in L_{n}\right\} \\
& +\sum\left\{p^{2 n-r-s} a_{j 1} \cdots a_{j 2^{r}} c_{i 1} \cdots c_{i 2^{s}}: 0 \leq r+s<2 n, j \in K_{r}, i \in L_{s}\right\} \\
= & 0,
\end{aligned}
$$

i.e. $B \in \Omega(p)$. 
Hence $\mathfrak{B}_{n+1} \leq \mathfrak{Q}(p) \cdot \mathfrak{B}_{n}$, and by induction, the lemma is proved.

Lemma 7. If $\mathfrak{U}$ contains $Q_{p}$ for some $p$, then $\mathfrak{U} \cdot \mathfrak{U}>\mathfrak{U}$.

If $\mathfrak{U}$ contains $Q_{p}$, then as $\mathfrak{I}(p)$ is a minimal variety [19], we have $\mathfrak{U} \geq \mathfrak{Q}(p)$. If $\mathfrak{U} \cdot \mathfrak{U}=\mathfrak{U}$, then $\mathfrak{U}=\mathfrak{U} \cdot \mathfrak{U} \geq \mathfrak{l}(p) \cdot \mathfrak{l}(p)$, and by induction $\mathfrak{U} \geq \mathfrak{I}(p)^{n}$. Hence by Lemma $6, \mathfrak{U} \geq \mathfrak{B}_{n}$ for all $n \geq 1$.

Claim l. $c(\overline{\mathfrak{U}})=0$. As $c\left(\overline{\mathfrak{B}}_{n}\right)=p^{n}, c(\mathfrak{U})$ is divisible by $p^{n}$ for all $n \geq 1$, i.e., $c(\mathfrak{U})=0$, and $\delta(\mathfrak{U})>1$.

Claim 2. For any varieties $\mathbb{B}, \mathbb{B}, \delta(\mathbb{B} \cdot \mathfrak{W})=\delta(\mathbb{B}) \cdot \delta(\mathbb{B})$. $\mathbb{B} \cdot \mathbb{B}$ is determined by $V(W)$, where $V$ and $W$ are the $T$-ideals of all identities satisfied by $B$ and $W$ respectively. Claim 2 is immediate by using the comments after Proposition 3.

From Claims 1 and 2 we get a contradiction, and $\mathfrak{U} . \mathfrak{U}>\mathfrak{U}$.

5. Lemma 8. If $\mathfrak{U}$ does not contain $Q_{p}$ for any prime $p$, then $\delta(\mathfrak{U})=1$ (i.e., $c(\mathfrak{U})>0)$.

If $c(\mathfrak{U})=0$, the free $\mathfrak{U}$-ring on one generator contains $\{0, x, 2 x, \ldots\}$ which is infinite. The factor of this ring by the ideal generated by $x^{2}$ is proper, otherwise $x=x^{2} q(x)$ would be an identity in $\mathfrak{u}$, and hence $k x=0$ would be an identity in $\mathfrak{U}$ for some $k>0$. The factor ring satisfies $x y=0$, and hence has a factor isomorphic to $Q_{p}$ for some $p$.

Lemma 9. If $\mathfrak{U} \neq \mathbb{E}, \mathfrak{u}$ satisfies $p x=0, x+x^{2} g(x)=0$, where $g(x) \in Z_{p}[x]$, then $\mathfrak{U}$ is generated by a finite number of finite fields of characteristic $p$.

By a theorem of Herstein [7], every ring satisfying $x+x^{2} g(x)$ is commutative. Also the identity $x+x^{2} g(x)=0$ tells that the ring does not have any nonzero nilpotent elements. Hence [8] every such ring is a subdirect product of fields. Since rings of $\mathfrak{U}$ also satisfy $p x=0$, then all fields involved are of characteristic $p$. As degree $\left(x+x^{2} q(x)\right)$ is fixed for all elements these fields involved must be finite in number, and each of which is of finite order, since if $n_{1}<n_{2}<n_{3}<\ldots$ $\Pi\left\{G F\left(p, n_{i}\right): i \geq 1\right\}$ does not satisfy any identity of the type $x+x^{2} g(x)=0$ for any $g(x) \in Z_{p}[x]$.

6. Lemma 10. If $\mathfrak{U} \neq \mathbb{E}, \mathfrak{B} \neq \mathbb{E}, \mathfrak{U}$ satisfies $p x, x+x^{2} g(x)$, and $\mathfrak{B}$ satisfies $p x=0, x+x^{2} b(x)$ where $g(x), b(x) \in Z_{p}[x]$, then $\mathfrak{U} \cdot \mathfrak{B}=\mathfrak{U} \vee \mathfrak{B}=\mathfrak{B} \cdot \mathfrak{U}$.

Substituting identities of $\mathfrak{B}$ in identities of $\mathfrak{n}$, we get identities of $\mathfrak{U} \cdot \mathfrak{B}$ (Proposition 3). Hence

$$
p^{2} x=0, \quad\left(x+x^{2} b(x)\right)+\left(x+x^{2} b(x)\right)^{2} g\left(x+x^{2} b(x)\right)=x+x^{2} f(x)=0
$$


and

$$
p x+p^{2} x^{2} g(p x)=0
$$

are identities in $\mathfrak{U} \cdot \mathfrak{B}$, hence $\mathfrak{U} \cdot \mathfrak{B} \neq \mathbb{E}$ satisfies $p x=0$ and $x+x^{2} f(x)=0$.

Hence by Lemma 9 , every ring in $\mathfrak{U} . \mathfrak{B}$ is the subdirect sum of finite fields, and every finitely generated ring is finite and hence the direct product of finite fields of characteristic $p$, i.e. every finitely generated ring has 1 . If $C \in \mathfrak{U} . \mathfrak{B}$ is finitely generated, its ideal belonging to $\mathfrak{U}$ is finite, and hence has a unit that is a central idempotent (since $C$ is commutative). Hence $C \cong A \times B$ where $A \in \mathfrak{U}, B \in \mathfrak{B}$. As every variety is generated by its finitely generated members, the lemma is proved.

Lemma 11. If $c(\mathfrak{U})=p^{k}, \mathfrak{U} \neq \Subset$ and $\mathfrak{U}$ does not contain $Q_{p}$, then $k=1$ and $\mathfrak{U}$ satisfies $p x=0$ and $x+x^{2} g(x)=0$ for some $g(x) \in Z_{p}[x]$.

Let $\mathfrak{B}$ be the variety of all rings in $\mathfrak{U}$ satisfying $p x=0$. As $\mathfrak{U}$ does not contain $Q_{p}, \mathfrak{B}$ does not contain $Q_{p} \cdot \mathfrak{B}$ satisfies $p x=0$ and $x+x^{2} g(x)=0$ for some $g(x) \in Z_{p}[x], g(x) \neq 0$. Indeed, the free ring of $\mathfrak{B}$ in one generator is $x Z_{p}[x] / x q(x) Z_{p}[x]$, degree $q(x) \in Z_{p}[x]$ is $\geq 1$, otherwise the free $\bigotimes_{\text {-ring }}$ on one generator is either 0 or $x Z_{p}[x]$ contradicting $\mathfrak{U} \neq \notin$ and $\mathfrak{U}$ does not contain $Q_{p}$. Hence $x q(x)=0$ is an identity in $B$, i.e., $a x+x^{2} g(x)=0$ is an identity in $\mathfrak{B}, \alpha \neq 0$, otherwise $x Z_{p}[x] / x^{2} Z_{p}[x] \in \mathfrak{B}$, that is $Q_{p} \in \mathfrak{B}$.

If $A \in \mathfrak{U}$ then $p^{r} A$ is an ideal of $A, r=0,1,2, \ldots, k$, and $p^{r} A / p^{r+1} A \in \mathfrak{B}$, $r=0,1, \cdots, k-1$. Thus $\mathfrak{B} \leq \mathfrak{U} \leq \mathfrak{B}^{k}=\mathfrak{B}$ by Lemma 10 .

Lemma 12. If $c\left(\mathfrak{U}_{1}\right)=p^{k}, c\left(\mathfrak{U}_{2}\right)=p^{l}, c\left(\mathbb{B}_{1}\right)=q^{m}, c\left(\mathbb{B}_{2}\right)=q^{n}$, where $p, q$ are distinct primes, then

$$
\left(\mathfrak{U}_{1} \cdot \mathfrak{B}_{1}\right) \cdot\left(\mathfrak{U}_{2} \cdot \mathfrak{B}_{2}\right)=\left(\mathfrak{U}_{1} \cdot \mathfrak{U}_{2}\right) \cdot\left(\mathfrak{B}_{1} \cdot \mathfrak{B}_{2}\right) \cdot
$$

Let $C \in\left(\mathfrak{U}_{1} \cdot \mathfrak{B}_{1}\right) \cdot\left(\mathfrak{U}_{2} \cdot \mathfrak{B}_{2}\right)$. There is $A$, an ideal of $C$, such that $A \in \mathfrak{U}_{1} \cdot \mathfrak{B}_{1}$ and $B=C / A \in \mathfrak{U}_{2} \cdot \mathfrak{B}_{2}$.

$$
\operatorname{ch} A=p^{\alpha} q^{\beta}, \quad \operatorname{ch} B=p^{\gamma} q^{\delta}, \quad \operatorname{ch} C=p^{\lambda} q^{\mu} \text {. }
$$

Thus

$$
\begin{array}{ll}
A=A_{1} \times A_{2}, & A_{1} \in \mathfrak{U}_{1}, \quad A_{2} \in \mathbb{B}_{1}, \\
B=B_{1} \times B_{2}, \quad B_{1} \in \mathfrak{U}_{2}, \quad B_{2} \in \mathbb{B}_{2}, \quad C=C_{1} \times C_{2} .
\end{array}
$$

$C_{1}$ contains all elements of $C$ whose order is a power of $p$. Thus $C_{1} \supseteq A_{1}$, $C_{2} \supseteq A_{2}$, as $C_{2}$ contains all elements of $C$ of order a power of $q$. 


$$
\left(C_{1} / A_{1}\right) \times\left(C_{2} / A_{2}\right) \cong\left(C_{1} \times C_{2}\right) /\left(A_{1} \times A_{2}\right) \cong B_{1} \times B_{2}
$$

as every element of $C_{1} / A_{1}$ or of $B_{1}$ is of order a power of $p$ and every element of $C_{2} / A_{2}$ or $B_{2}$ is of order a power of $q$. Hence $C_{1} / A_{1} \cong B_{1}, C_{2} / A_{2} \cong B_{2}$, i.e., $C \in\left(\mathfrak{U}_{1} \cdot \mathfrak{u}_{2}\right) \cdot\left(\mathfrak{B}_{1} \cdot \mathfrak{B}_{2}\right)$.

Conversely if $C \in\left(\mathfrak{U}_{1} \cdot \mathfrak{U}_{2}\right) \cdot\left(\mathfrak{B}_{1} \cdot \mathfrak{B}_{2}\right)$, there is an ideal $A$ of $C$ such that $A \in \mathfrak{U}_{1} \cdot \mathfrak{U}_{2}$ whose factor $C / A \in \mathfrak{B}_{1} \cdot \mathfrak{B}_{2}$. But ch $A=p^{\alpha}$, ch $C / A=q^{\beta}$, hence $C \cong A \times(C / A)$, there are ideals $A_{1}$ of $A$ and $A_{2}$ of $B=C / A$ such that $A / A_{1} \epsilon$ $\mathfrak{U}_{2}, A_{1} \in \mathfrak{U}_{1}, B / A_{2} \in \mathfrak{B}_{2}, A_{2} \in \mathfrak{B}_{1}$. Hence $A_{1} \times A_{2}$ is an ideal of $C$, belonging to $\mathfrak{U}_{1} \cdot \mathfrak{B}_{1}$ and $C /\left(A_{1} \times A_{2}\right) \cong(A \times B) /\left(A_{1} \times A_{2}\right) \cong\left(A / A_{1}\right) \times\left(B / A_{2}\right) \in \mathfrak{U}_{2} \cdot \mathfrak{B}_{2}$.

Lemma 13. If $\mathfrak{U}$ does not contain $Q_{p}$ for any prime $p$ and $\mathfrak{U} \neq \mathbb{E}$, then $\mathfrak{U}=\mathfrak{U}_{1} \times \mathfrak{U}_{2} \times \cdots \times \mathfrak{U}_{k}$, where $\mathfrak{U}_{i} \neq \mathbb{E}$ satisfies $p_{i} x=0, x+x^{2} g_{i}(x)=0$ for some $\boldsymbol{g}_{i}(x) \in Z_{p_{i}}(x)$, where $p_{1}, \cdots, p_{k}$ are $k$ distinct primes, $k \geq 1, c(\mathfrak{U})=p_{1} p_{2} \cdots p_{k}$.

If $\mathfrak{U}$ does not contain $Q_{p}$ for any $p, c(\mathfrak{U})=n>0$ (by Lemma 8). Let $n=p_{1}^{r_{1}} \cdots p_{k}^{r_{k}}$ be the prime decomposition of $n, r_{1}, \cdots, r_{k}>0$. Hence, $\mathfrak{U}=\mathfrak{U}_{1}$ $\times \mathfrak{U}_{2} \times \cdots \times \mathfrak{U}_{k}$, where $\mathfrak{U}_{i}$ is the family of rings of $\mathfrak{U}$ satisfying $p_{i}^{r_{i}} x=0$. Thus $\mathfrak{U}_{i}$ does not contain $Q_{p_{i}}$, and hence (by Lemma 11) $r_{i}=1$ and $\mathfrak{U}_{i}$ satisfies $x+x^{2} g_{i}(x)$ for some $g_{i}(x) \in Z_{p}[x], g_{i}(x) \neq 0$.

7. By the previous lemmas, if $\mathfrak{U}$ does not contain $Q_{p}$ for any prime $p, \mathfrak{U}$ is the join of a finite number of varieties of the type $p x=0, x+x^{2} g(x)=0$, $g(x) \neq 0, g(x) \in Z_{p}[x]$. Hence, $\mathfrak{U}$ does not contain any $Q_{p}$ implies that $\mathfrak{U}$ is generated by a finite number of finite fields, i.e., $\mathfrak{U}=\bigvee\{\mathfrak{P}(p, n): p \in K, n \in L\}$, where $K$ is a finite set of primes and $L$ is a finite set of positive integers.

If $\mathfrak{U}=\bigvee \mathfrak{i} \mathfrak{B}(p, n): p \in K, n \in L\}$, then $\mathfrak{U}=\mathfrak{U}_{1} \cdot\left(\mathfrak{U}_{2} \cdot\left(\ldots\left(\mathfrak{U}_{k}\right)\right)\right.$, where $\mathfrak{u}_{i}$ is the variety of all rings of $\mathfrak{U}$ of characteristic $p_{i}$; they satisfy also $\boldsymbol{x}+x^{2} g_{i}(x)=0$, and hence $\mathfrak{u}_{i} \cdot \mathfrak{u}_{i}=\mathfrak{U}_{i} \vee \mathfrak{u}_{i}=\mathfrak{U}_{i}$ (by Lemma 10), By Lemma 12 and induction on $k$, we get $\mathfrak{U} \cdot \mathfrak{U}=\mathfrak{U}$.

If $\mathfrak{U} \neq \mathfrak{S}$ and $\mathfrak{U} \cdot \mathfrak{U}=\mathfrak{U}$, then $\mathfrak{U}$ does not contain $Q_{p}$ for any $p$ (by Lemma 7 ). This concludes the proof of Theorem 5 .

Theorem 14. Let $H$ be the sct of all idempotent varieties distinct from $\supseteqq$. $H$ is a subalgebra of $\langle G ; \cdot, \wedge, \vee\rangle$, and on $H$, the product coincides with the join. $H$ is an ideal of the lattice $\langle G ; \wedge, \vee\rangle$, isomorphic to the lattice of all finite ideals of the poset $\{(p, n): p$ is prime, $n \geq 1\},(p, n) \leq(q, m) \Leftrightarrow p=q$ and $n \mid m$. The complement of $H$ in $G$ is an ideal of the groupoid $\langle G,\rangle.\langle\langle,$.$\rangle is an ideal$ extension of the complement of $H$ by the lattice $H$.

8. As an application of Theorem 5 , we will determine all attainable identities on $?$. 
Let $U \subseteq F$, and let $\bar{U}$ be the $T$-ideal of $F$ generated by $U$.

Definition 3 [17], [18]. A ring $R$ is said to be $U$-decomposable if $\bar{U}(R) \neq R$ and $U$-indecomposable if $\bar{U}(R)=R$. If $\Omega$ is a class of rings such that every $R \in \Omega$. is $U$-decomposable and $\bar{U}(R)$ is $U$-indecomposable, $U$ is said to be attainable on $R$.

In [18] it is shown that $\{x y=y x\},\left\{x=x^{p}, p x=0\right\}$ are not attainable on $\bigcirc$.

Theorem 15. No family of identities is attainable on $\bigcirc$ unless it is equivalent to $x=x$ or $x=y$.

If $\mathfrak{U}$ is attainable on $D$, then $\mathfrak{U}$ is idempotent [9], and if $U$ does not imply $x=y, \mathfrak{U} \neq D$, then $\mathfrak{U}$ is the product of a finite number of $\mathfrak{B}(p, n)$, i.e., $\mathfrak{u}=\mathfrak{U}_{1} \cdot\left(\mathfrak{U}_{2}\left(\ldots\left(\mathfrak{U}_{k}\right) \ldots\right)\right)$, where $\mathfrak{U}_{i}$ is the product of $\mathfrak{B}\left(p_{i}, n_{i j}\right)$ for a fixed $p_{i}, p_{1}, \cdots, p_{k}$ are distinct primes.

$$
U\left(Z_{p}[x]\right)=U_{1}\left(U_{2}\left(\cdots\left(U_{k}\left(Z_{p_{1}}[x]\right)\right) \cdots\right)\right)=U_{1}\left(Z_{p_{1}}[x]\right),
$$

since $U_{2}\left(\cdots\left(U_{k}\left(Z_{p}[x]\right)\right) \cdots\right) \supseteq p_{2} \cdots p_{k} Z_{p_{1}}[x]=Z_{p_{1}}[x]$, and the same is true for any ring of characteristic $p_{1}^{r}$. Thus

$$
U\left(U\left(Z_{p}[x]\right)\right)=U_{1}\left(U_{2}\left(\ldots\left(U_{k}\left(U_{1}\left(Z_{p}[x]\right)\right) \ldots\right)\right)\right)=U_{1}\left(U_{1}\left(Z_{p}[x]\right)\right)
$$

i.e., the proof is reduced to the case $\mathfrak{U}=\Re\left(p, n_{1}\right) \cdot\left(\Re\left(p, n_{2}\right)\left(\ldots\left(\Re\left(p, n_{k}\right)\right) \ldots\right)\right)$.

$U\left(Z_{p}[x]\right)$ is principal, and hence generated by $q(x)$, where $q(x)$ is divisible by all $x-x^{p^{n_{i}}}, 1 \leq i \leq k$, i.e., $\operatorname{deg} q(x)>1$, i.e., $K=U\left(Z_{p}[x]\right)=q(x) Z_{p}[x] . U(K)$ is the ideal of $K$ generated by the values of polynomials of $U$ in $K . U(K) \subseteq$ $P\left(p, n_{1}\right)(K)=L . f \in L$ iff

$$
\begin{aligned}
& f=\sum\left\{\left[q(x) f_{n}(x)-\left(q(x) f_{n}(x)\right)^{N}\right] q(x)^{r} g_{n r}(x):\right. \\
& \left.\quad f_{n}(x), g_{n r}(x) \in Z_{p}[x], r \geq 0, r=0 \Rightarrow g_{n r}=1\right\}, \quad N=p^{n} .
\end{aligned}
$$

Thus $f=f_{1}+f_{2}+f_{3}$;

$$
\begin{aligned}
& f_{1}=a_{0}\left(q(x)-q(x)^{N}\right), \\
& f_{2}=\sum\left\{a_{n}\left[q(x) x^{n}-q(x)^{N} x^{n N}\right]: n \geq 1\right\}, \\
& f_{3}=\sum\left\{b_{n r s}\left[q(x)^{1+r} x^{n+s}-q(x)^{N+r} x^{n N+s}\right], n \geq 0, r>0, s>0\right\}, \quad a_{0}, a_{n}, b_{n r s} \in Z_{p} .
\end{aligned}
$$

$f_{2}=q(x) x^{\alpha} g(x), g(x)$ is not divisible by $q(x), f_{3}=q(x)^{\beta} x^{\gamma} b(x), b(x)$ is not divisible by $q(x), a>0, \beta>1, \gamma>0$. If $q(x) \in L$ then

$$
q(x)=a_{0}\left(q(x)-q(x)^{N}\right)+q(x) x^{\alpha} g(x)+q(x)^{\beta} x^{\gamma} b(x) ;
$$


hence $a_{0}=1$ and $q(x) x^{\alpha} g(x)=q(x)^{N}-q(x)^{\beta} x^{\gamma} b(x)$ i.e., $g(x)$ is divisible by $q(x)$, i.e., $L \neq K$.

This proves that $K$ is not $U$-indecomposable, and hence $\mathfrak{U}$ is not attainable on $\bigcirc$ which concludes the proof of Theorem 15. The methods of this paper can be modified to get the same attainability result for commutative rings.

\section{REFERENCES}

1. G. Birkhoff, Lattice theory, 3rd ed., Amer. Math. Soc. Colloq. Publ., vol. 25, Amer. Math. Soc., Providence, R. I., 1967. MR 37 \#2638.

2. P. M. Cohn, Universal algebra, Harper \& Row, New York, 1965. MR 31 \#224.

3. - Free rings and their relations, Academic Press, London and New York, 1971.

4. C. J. Everett, An extension theory for rings, Amer. J. Math. 64 (1942), 363-370. MR 4,69.

5. J. Goldman and S. Kass, Linearization in rings and algebras, Amer. Math. Monthly 76 (1969), 348-355. MR 39 \#2821. $\# 1320$.

6. G. Grätzer, Universal algebra, Van Nostrand, Princeton, N.J., 1968. MR 40

7. I. N. Herstein, The structure of a certain class of rings, Amer. J. Math. 75 (1953), 864-871. MR 15, 392.

8. N. H. McCoy, Rings and ideals, Carus Monographs Series, no. 8, Open Court, La Salle, Ill., 1948. MR 10, 96.

9. A. I. Mal'cev, Multiplication of classes of algebraic systems, Sibirsk. Math. Ž. 8

(1967), 364-365 = Siberian Math. J. 2 (1967), 245-267. MR 35 \#4140.

10. - Algebraic systems, "Nauka", Moscow, 1970; English transl., Die

Grundlehren der math. Wissenschaften, Band 192, Springer-Verlag, Berlin and New York, 1973.

11. B. H. Neumann, Hanna Neumann and P. M. Neumann, Wreath products and varieties of groups, Math. Z. 80 (1962), 44-62. MR 25 \#5102.

12. Hanna Neumann, On varieties of groups and their associated near-rings, Math. $Z$. 65 (1956), 36-69. MR 17, 1183.

13. J. M. Osborn, Varieties of algebras, Advances in Math. 8 (1972), 163-369. MR $44 \# 6775$.

14. V. A. Parfenov, Varieties of Lie algebras, Algebra i Logika 6 (1967), no. 4, 6173. (Russian) MR 37 \#2825.

15. O. Schreier, Über die Erweiterung von Gruppen. I, II, Monatsh. Math. Phys. 34 (1926), 165-180.

16. A. L. Šmelkin, The semigroup of group manifolds, Dokl. Akad. Nauk SSSR 149 (1963), 543-545 = So viet Math. Dokl. 4 (1963), 449-451. MR 27 \#1524.

17. T. Tamura, Attainability of systems of identities on semigroups, J. Algebra 3 (1966), 261-276. MR $32 \# 7668$.

18. T. Tamura and F. M. Yaqub, Examples related to attainability of identities on lattices and rings, Math. Japon. 10 (1965), 35-39. MR 33 \#79.

19. A. Tarski, Equationally complete rings and relation algebras, Nederl. Akad. Wetensch. Proc. Ser. A 59 = Indag. Math. 18 (1956), 39-46. MR 18, 636.

DEPARTMENT OF MATHEMATICS, UNIVERSITY OF SOUTHWESTERN LOUISIANA, LAFAYETTE, LOUISIANA 70501 